\title{
A novel germline variant in the DOT1L gene co-segregating in a Dutch family with a history of melanoma Catarina Salgado ${ }^{a}$, Eliza Mari Kwesi-Maliepaard ${ }^{b}$, Aart G. Jochemsen ${ }^{c}$, Mijke Visser $^{\mathrm{a}}$, Mark Harland ${ }^{\mathrm{d}}$, Fred van Leeuwen ${ }^{\mathrm{b}}$, Remco van Doorn ${ }^{\mathrm{a}}$ and Nelleke Gruis ${ }^{a}$
}

\begin{abstract}
A proportion of patients diagnosed with melanoma has a positive family history. Despite increasing knowledge on the genes responsible for familial clustering, the genetic basis in the majority of the families with an inherited predisposition to melanoma remains to be clarified. To identify novel melanoma-susceptibility genes, we applied whole-exome sequencing on DNA from two members of a family with four melanoma cases, not explained by established high penetrance melanoma-susceptibility genes. Whole-exome sequencing identified 10 rare, co-segregating, predicted deleterious missense gene variants. Subsequent co-segregation analysis revealed that only variants in the DOT1L (R409H) and the SLCO4C1 (P597A) genes were present in the other two affected members of this family. DOT1L is a methyltransferase that methylates histone $\mathrm{H} 3$ lysine 79 (H3K79). It is involved in maintenance of genomic stability, since mutations in the DOT1L gene have been previously reported to compromise the removal of ultraviolet photoproducts in ultraviolet-irradiated melanocytes, thereby enhancing malignant transformation. We hypothesized that the presence of DOT1L R409H variant might be associated with an increased risk of melanoma, since we found co-segregation of the DOT1L mutation in all four
\end{abstract}

melanoma-affected family members. However, this missense variant did neither lead to detectable lossof-heterozygosity nor reduction of histone methyltransferase activity in melanoma samples from mutation carriers nor altered ultraviolet-survival of mouse embryonic stem cells containing an engineered homozygous DOT1L R409H mutation. Although functional analysis of this rare co-segregating variant did not reveal compromised histone methyltransferase activity and ultraviolet exposure sensitivity, the role of DOT1L as melanoma susceptibility gene deserves further study. Melanoma Res 29:582-589 Copyright (C) 2019 Wolters Kluwer Health, Inc. All rights reserved.

Melanoma Research 2019, 29:582-589

Keywords: DOT1L, familial melanoma, histone methyltransferase, whole-exome sequencing

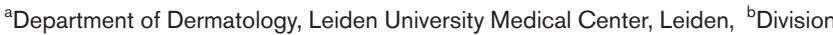
of Gene Regulation, Netherlands Cancer Institute, Amsterdam, 'Department of Cell and Chemical Biology, Leiden University Medical Center, Leiden, The Netherlands and ${ }^{\mathrm{d}}$ Section of Epidemiology and Biostatistics, Leeds Institute of Medical Research at St James's, University of Leeds, Leeds, UK

Correspondence to Catarina Salgado, MSc, Leiden University Medical Center, PO Box 9600, 2300 RC Leiden, The Netherlands

Tel: +31 71 5266378; e-mail: C.M.A.Salgado@lumc.nl

Received 20 March 2019 Accepted 19 July 2019

offered to the patients [3]. Exome-wide sequencing approaches can be valuable in the identification of putative new-melanoma susceptibility genes [4].

In the present study, we describe a Dutch family of which four family members were diagnosed with melanoma. Whole-exome sequencing (WES) of DNA from two family members identified a new germline missense variant c.G1226A:p.R409H in the DOT1L gene, that co-segregated with melanoma in all four affected family members. DOT1L is the unique histone methyltransferase responsible for methylating the nucleosome core on lysine 79 of histone H3 (H3K79) [5,6]. The observed DOT1L variant appeared to be the most promising pathogenic variant since recently loss of DOT1L (by silencing or mutation) has been reported to promote melanomagenesis in a pre-clinical mouse model upon ultraviolet (UV) radiation [7]. The role of DOT1L in DNA damage repair pathway involves the transcriptional recovery through
Supplemental Digital Content is available for this article. Direct URL citations appear in the printed text and are provided in the HTML and PDF versions of this article on the journal's website, www.melanomaresearch.com. 
reactivation of RNA Pol II in mouse-derived cell lines [8] and the recruitment of $X P C$ for an efficient nucleotide excision repair in melanocytes and cell lines derived from human melanoma, thereby protecting melanocytes from the UV-induced transition to melanoma [7].

We hypothesized that in the family under study, the $\mathrm{R} 409 \mathrm{H}$ DOT1L variant represents a loss of function mutation which diminishes the protective role of DOT1L enhancing melanoma development.

\section{Methods}

\section{Patients}

DNA from members of a Dutch family with four family members affected with melanoma was isolated from whole blood samples, a primary tumor and a brain metastasis. The study was approved by the Leiden University Medical Center institutional ethical committee (LUMC, P00.117). The affected family members were tested negative for variants in the high penetrance genes CDKN2A and CDK4 and cases II.2 and III.3 were subjected to WES (Fig. 1).

\section{Whole-exome sequencing}

Whole-exome sequencing was performed using Agilent All-exon capture baits (Agilent Technologies, Santa Clara, California, USA) and sequenced on the Illumina platform at Sanger Institute, Cambridge, UK. The bioinformatics analysis and subsequent filtering steps were performed at Sanger Institute and later confirmed by our in-house bioinformatics pipeline. Briefly, the reads were aligned to the human genome build hg19 using BurrowsWheeler Alignment tool [9]. To pass the filtering steps

Fig. 1

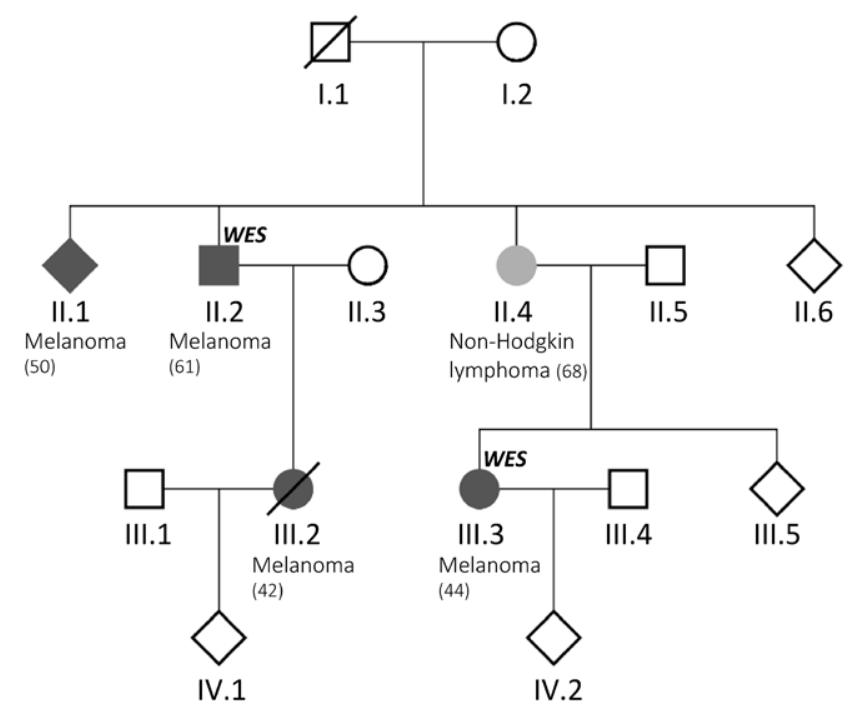

Family pedigree. The melanoma affected members are in dark gray color (II.1, II.2, III.2, III.3). The age of diagnosis is indicated in brackets. The melanoma cases subjected to whole-exome sequencing are indicated by an 'WES'. the variants needed to have a high-quality score $(>30)$, to have high coverage $(>40 \times)$, to be a nonsynonymous single nucleotide variant, to be heterozygous present in both samples, to have low ExAC frequency $(<0.001)$, and needed to be predicted as deleterious and damaging by Polyphen and SIFT. Variants which did not fulfill all the filtering criteria were excluded, resulting in a list of 10 variants of interest (Table 1).

\section{Co-segregation analysis}

All 10 variants were confirmed by Sanger sequencing using DNA from the two family members subjected to WES (II.2 and III.3, see Figure, Supplemental Digital Content 1, http://links.lww.com/MR/A177). Briefly, 20-100 ng of DNA was amplified through a touchdown PCR using the Platinum Taq DNA Polymerase following the manufacturer's instructions (Invitrogen, Carlsbad, California, USA). The PCR product was cleaned-up using the NucleoSpin Gel and PCR Clean-Up (Macherey-Nagel GmbH \& Co. KG, Düren, Germany) according to manufacturer's instructions. Then, Sanger sequencing was performed using 20-50 ng of purified DNA mixed with $1 \mu \mathrm{l}$ of $10 \mu \mathrm{M}$ of sequencing primer and nuclease-free water (B. Braun, Melsungen, Germany) up to $10 \mu$ l. Later on, we used the same approach to evaluate the co-segregation of these variants with melanoma in all affected family members (see Figure, Supplemental Digital Content 2, http://links.lww.com/MR/A178 and Fig. 2)

\section{Loss of heterozygosity analysis}

Loss of heterozygosity ( $\mathrm{LOH}$ ) was assessed by droplet digital PCR (ddPCR). Tumors from two family members were examined: a formalin-fixed paraffin-embedded (FFPE)-derived primary melanoma biopsy from II.1 and a brain metastasis from III.2 (Fig. 2). The DNA extraction was performed using Tissue Preparation System (Siemens Healthcare GmbH, Erlangen, Germany) at the Department of Pathology, LUMC. Briefly, $10 \mathrm{ng}$ of DNA was combined with 1X ddPGR Mut Assay DOT1L R409H (dHsaMDS130625855; Bio-Rad Laboratories, Inc., Hercules, California, USA), 1X ddPCR supermix for probes (no dUTP) (Bio-Rad), 1 U/pl MseI restriction enzyme [New England Biolabs, Inc. (NEB), Ipswich,

Table 1 Germline variants identified by whole exome sequencing shared by two affected family members of a four case Dutch melanoma family

\begin{tabular}{|c|c|c|c|}
\hline Gene & Location & Variant & Amino acid substitution \\
\hline SLCO4C1 & Chr5: $101582978-101582978$ & $G>C$ & P597A \\
\hline PEX6 & Chr6: $42932102-42932102$ & $G>A$ & R972C; R884C \\
\hline$F B X L 13$ & Chr7: $102462622-102462622$ & $G>A$ & S583L; S600L; S628L \\
\hline NAIF1 & Chr9: $130825802-130825802$ & $\mathrm{G}>\mathrm{A}$ & R297C \\
\hline LAMC3 & Chr9: $133914340-133914340$ & $\mathrm{C}>\mathrm{T}$ & R356C \\
\hline $\mathrm{C} / \mathrm{T}$ & Chr12: $120152035-120152035$ & $C>T$ & V1383M; V1425M \\
\hline FREM2 & Chr13: 39433637-39433637 & $\mathrm{C}>\mathrm{T}$ & R2477W \\
\hline DOT1L & Chr19: 2210729-2210729 & $G>A$ & $\mathrm{R} 409 \mathrm{H}$ \\
\hline FUT1 & Chr19: $49253896-49253896$ & $C>A$ & V215F \\
\hline UMODL1 & Chr21: $43508479-43508479$ & $G>A$ & V155M; V227M \\
\hline
\end{tabular}




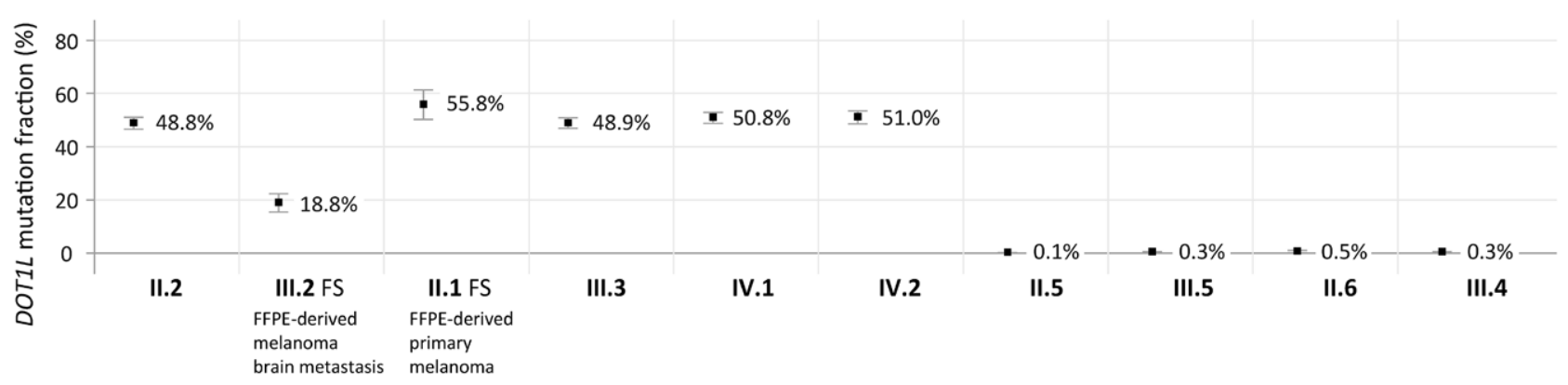

Droplet digital PCR results showing the DOT1L mutation fraction in DNA samples extracted from whole blood samples [II.2, III.3 (both subjected to WES) and IV.1 and IV.2], a primary melanoma (II.1 FS) and a brain metastasis (III.2 FS). The mutation fraction is around $50 \%$ in mutationcarriers and around $0 \%$ in wild-type family members (II.5, II.6, III.4, III.5), as control samples. In a full section (III.2 FS) of the brain metastasis, a mutation fraction of $\sim 20 \%$ was found. The primary melanoma (II.1 FS) is mutated for DOT1L; however, no LOH was observed. LOH, loss of heterozygosity; WES, whole-exome sequencing.

Massachusetts, USA] diluted in its own buffer CutSmart (NEB) and nuclease-free water (B. Braun) up to $22 \mu 1$. To generate droplets the Automated Droplet Generator (Bio-Rad Laboratories, Inc.) was used, followed by the PCR using the cycling conditions for Bio-Rad's C1000 Touch Thermal Cycler (Bio-Rad Laboratories, Inc.) with an annealing temperature of $55^{\circ} \mathrm{C}$. The number of droplets was determined by the QX200 Droplet Reader (BioRad Laboratories, Inc.) and analysed using QuantaSoft version 1.7.4.0917 (Bio-Rad Laboratories, Inc.).

\section{Immunohistochemistry}

For immunohistochemistry (IHC, see Figure, Supplemental Digital Content 3, http://links.lww.com/MR/ A179), sections from FFPE-derived primary melanomas from II.1 and brain metastasis from III.2, along with EAF (ethanol, acetic acid, formol saline) fixed and paraffin-embedded thymus tissues from mice with conditional deletion of Dot1L [10] were pre-incubated with goat serum (Dako; Agilent Technologies) for 30 minutes and then incubated overnight with H3K79me2 antibody (1:8000 dilution, RRID:AB_1587126 [11]) followed by incubation with Dako EnVision+ System HRP labeled polymer anti-rabbit (Agilent Technologies) for 30 minutes. The slides were washed with PBS, incubated with Dako 3,3'-diaminobenzidine substrate chromogen system (dilution 1:50; Agilent Technologies), and counterstained with hematoxylin (Merck KGaA, Darmstadt, Germany).

\section{Cell lines generation}

IB10 wild-type mouse embryonic stem cells (mESCs) were used to engineer site-specific mutations using CRISPR/Cas9 to generate mESC lines expressing DOT1L R409H or the catalytic site mutant DOT1L G165R according to the protocol described by Harmsen $e t$ al. [12] IB10 mESCs were cultured on a feeder layer of irradiated murine embryonic fibroblasts in complete medium containing GMEM-BHK12 (Gibco/Thermo Fisher Scientific,
Waltham, Massachusetts, USA), $100 \mathrm{mM}$ Sodium Pyrovate (Gibco), non-essential amino acids (Gibco) and 10\% ES cell certified serum (HyClone/Thermo Fisher Scientific). This was complemented with $0.1 \mu \mathrm{M} \beta$-mercaptoethanol (Sigma-Aldrich, St. Louis, Missouri, USA) and mouse recombinant leukemia inhibitor factor (Merck KGaA). For transfection, cells were grown on gelatin-coated plates in $60 \%$ Buffalo Rat Liver (BRL) medium (150 ml BRLconditioned medium $+100 \mathrm{ml}$ complete medium). Cells were incubated at $5 \% \mathrm{CO}_{2}$, at $37^{\circ} \mathrm{C}$.

Oligonucleotides encoding the gRNAs are in Supplemental Digital Content 4, http://links.lww.com/MR/ A180. Single strand homology-directed repair (HDR) templates are in Supplemental Digital Content 5, http:// links.lww.com/MR/A181. The repair templates were purchased from Sigma-Aldrich, all other oligonucleotides from IDT (Leuven, Belgium). The gRNAs were cloned into the px330.pgkpuro vector (a gift from Hein te Riele). A mixture of $0.1 \mu \mathrm{g}$ CRISPR/Cas 9 vector and $0.4 \mu \mathrm{g}$ HDR template in optiMEM (Gibco) with $1.25 \mu \mathrm{l}$ TransIT LT1 (Mirus Bio LLG, Madison, Wisconsin, USA) was incubated for 15-20 minutes at room temperature and added to the cells. The next day cells were replated in $60 \% \mathrm{BRL}$ medium containing $3.6 \mu \mathrm{g} / \mathrm{ml}$ puromycin. Two days later the medium was replaced with medium without puromycin. Cells were then sparsely seeded to grow single clones. After 1 week, single clones were selected and genomic DNA was isolated to validate the mutations, which also introduced restriction sites. The regions containing the R409H and G165R mutations were amplified using MyTaq Redmix (GC-Biotech B.V., Alphen aan den Rijn, The Netherlands) and the following primers: R409H-5"TGCCTCAGCCTATGGTCTTGT and 5"TGGCACATGGCAGAGTCCCATA, for G165R5'ACTACACAGCCCATGAAGCTGA and 5"TGGTTA AGCAGCCACAACCCA. The PCR product containing the $\mathrm{R} 409 \mathrm{H}$ region was digested with MIcI (Thermo 
Fisher Scientific) directly after amplification. PCR products containing the $\mathrm{G} 165 \mathrm{R}$ region were purified using the QIAquick PCR purification kit (Qiagen, Hilden, Germany) according to the manufacturers protocol and then digested with BauI (Thermo Fisher Scientific). Clones that showed the expected digestion pattern were further validated using Sanger sequencing. For UV-survival assays three clones with the R409H mutation were selected and two clones that had the G165R mutation, of which one had a homozygous G165R and one clone with a heterozygous G165R mutation and one nucleotide deletion causing a frame-shift in DOT1L.

\section{Ultraviolet-survival assay}

To assess UV sensitivity, we performed a colony formation assay upon UV-C exposure in wild-type and CRISPR/Cas9 engineered DOT1L mutant mESCs. For UV-survival assays mESCs were cultured in $60 \% \mathrm{BRL}$ conditioned medium. One thousand cells were plated in a $10-\mathrm{cm}$ dish and grown overnight. The next day cells were washed with PBS and exposed to UV-C irradiation (254 nm, UV-C irradiation chamber; Dr Gröbel UV-Elektronik, GmgH, Germany; dose range: 0.5, 1, 2, 4, $8 \mathrm{~J} / \mathrm{m}^{2}$ ). After 8 days of incubation the colonies were fixed and stained using Leishman's eosin methylene blue solution modified (Merck KGaA). Colonies were counted with the ColCount (Oxford Optronix Ltd., Abingdon, UK).

\section{Western blot}

Murine ESCs were grown in feeder-free conditions in serum-free ES cell medium containing neurobasal medium (Thermo Fisher Scientific), DMEM/F12 (Thermo Fisher Scientific), N2 supplement (Thermo Fisher Scientific), B27 (Thermo Fisher Scientific) and BSA (Thermo Fisher Scientific) supplemented with GSK inhibitor CHIR99021 (BioConnect B.V., Huissen, The Netherlands) and MEK1\&2 inhibitor PD0325901 (BioConnect) and cell pellets were frozen. Lysates were made using 1X SDS sample buffer $(50 \mathrm{mM}$ Tris-HCl pH 6.8, 2\% SDS, $10 \%$ glycerol) and sonicated. Samples were boiled for 5 minutes in $5 \mathrm{X}$ SDS-sample buffer (250 mM Tris-HCl pH 6.8, 10\% SDS, 50\% glycerol, 0.5 M DTT, $0.5 \%$ bromophenol blue) and separated on a $16 \%$ polyacrylamide gel. Separated proteins were transferred on a $0.45 \mu \mathrm{m}$ nitrocellulose membrane for 1 hour. Membranes were blocked using 2\% Nutrilon (Nutricia/ Danone, Schiphol, The Netherlands) in Tris-buffered saline containing $0.05 \%$ Tween-20 (TBST) for 1 hour and incubated overnight with anti-H3K79me1 (RRID: AB_2631105), anti-H3K79me2 (RRID: AB_2631106) or anti-H3 (ab1791; Abcam, Cambridge, UK). All antibodies were diluted 1:100 in TBST containing 2\% Nutrilon. After incubation, the membranes were washed three times with TBST and incubated with IRDye $800 \mathrm{CW}$ goat anti-Rabbit igg (1:10 000, Li-COR (RRID: AB_621843)) for 45 minutes in TBST with $2 \%$ Nutrilon. Then, the membranes were washed three times with TBST and once in PBS and scanned using an LI-COR Odyssey IR Imager (LI-COR Biosciences, Lincoln, Nebraska, USA). Images were analysed using Image studio 2.0 (LI-COR Biosciences).

\section{Results}

$\mathrm{R} 409 \mathrm{H}$, a novel germline variant of the DOT1L gene identified in a four case melanoma family

Upon WES of the DNA from two melanoma cases (II.2 and III.3, Fig. 1), 4892 heterozygote variants were found. The bioinformatics analysis encompassed the alignment to genome build hg19 and the above-mentioned filtering criteria. Frameshift and truncating variants were found but did not pass the ExAC filter. Only 10 rare, co-segregating, predicted deleterious missense variants in the genes SLCO4C1, PEX6, FBXL13, NAIF1, LAMC3, CIT, FREM2, DOT1L, FUT1, UMODL1 met our criteria (Table 1). The presence of these 10 germline variants in the two cases (II.2 and III.3) subjected to WES was confirmed by Sanger sequencing in DNA derived from blood leukocytes (see Figure, Supplemental Digital Content 1, http://links.lww.com/MR/A177). Subsequently, co-segregation of the variants was evaluated in other family members for whom DNA was available (II.1, 2, 5, 6; III. 2, 3, 4, 5; IV. 1, 2; data not shown).

Only two out of 10 variants co-segregated with melanoma in all four affected relatives: c.C1789G:p.P597A in SLCO4C1 gene and c.G1226A:p.R409H in DOT1L gene (see Figure, Supplemental Digital Content 2, http:// links.lww.com/MR/A178 and Fig. 2). The SLCO4C1 gene encodes for a member of the organic anion transporting polypeptide family. Human SLCO4C1 is involved in the membrane transport of cardiac glycosides, thyroid hormones, bile acids and many other compounds [13]. However, a putative function for SLCO4C1 in cancer development is unclear. Only two studies describe SLCO4C1 mutation or silencing in head and neck cancers, affecting the platinum uptake and clearance [14,15]. SLCO4C1 is not expressed in melanocytes and melanomas according to publicly available databases $[16,17]$. Taken together, these reasons appear to exclude SLCO4C1 as a candidate susceptibility gene for the family under investigation.

DOT1L is the unique histone methyltransferase responsible for methylating the nucleosome core on H3K79. Based on the function of the DOT1L gene in UV-induced DNA damage repair and its reported role in melanoma development, we considered the DOT1L gene variant a strong candidate responsible for melanoma susceptibility in this family. Additional rare and possibly deleterious variants were found in four sporadic and familial melanoma cases from the UK (Table 2). Moreover, the 19p13.3 locus, containing DOT1L gene, has been shown to be frequently deleted in metastatic melanoma cases [18]. 
Table 2 Additional DOT1L variants found in familial and sporadic melanoma cases from the UK

\begin{tabular}{|c|c|c|c|c|c|c|}
\hline Location & Variant & $\begin{array}{l}\text { Amino acid } \\
\text { substitution }\end{array}$ & Polyphen & SIFT & $\begin{array}{l}\text { Allele frequency } \\
\quad \text { (gnomAD) }\end{array}$ & Familial history vs. sporadic \\
\hline Chr19: 2226478 & $G>A$ & G1320R & Possibly damaging & Deleterious & $1.918 \mathrm{e}-5$ & $\begin{array}{l}\text { Melanoma family with } 2 \text { cases of } \\
\text { melanoma and multiple primaries }\end{array}$ \\
\hline Chr19: 2191090 & $A>T$ & Y115F & Probably damaging & Deleterious & 0 & \\
\hline Chr19: 2226839 & $G>C$ & S1440T & Benign & Deleterious & 0 & Sporadic case, with early onset \\
\hline Chr19: 2213960 & $C>T$ & A591V & Benign & Tolerated & 0 & $\begin{array}{l}\text { Melanoma family with } 3 \text { cases of } \\
\text { melanoma and multiple primaries }\end{array}$ \\
\hline Chr19: 2217838 & $\mathrm{~T}>\mathrm{C}$ & L871P & Probably damaging & Tolerated & 0 & Sporadic case, with early onset \\
\hline
\end{tabular}

\section{Absence of loss of heterozygosity and altered methyltransferase capacity in tumor samples from melanoma-affected family members}

First, we assessed LOH of DOT1L p.R409H by Sanger sequencing and ddPCR analysis in a FFPE-derived primary melanoma biopsy from individual II.1 and melanoma brain metastasis from individual III.2. In the ddPCR result, the mutation fraction is about $50 \%$ in mutation-carriers [II.2, III.3 (both subjected to WES) and IV.1 and IV.2, the youngest family members who did not develop melanoma yet] and close to $0 \%$ in wild-type family members (II.5, II.6, III.4 and III.5). We observed a low mutation fraction in the metastasis from III.2 $(\sim 20 \%)$ and a mutation fraction of about $56 \%$ in the primary tumor from II.1 (Fig. 2). These numbers show an absence of $\mathrm{LOH}$ in the primary tumor and the brain metastasis.

Then, we checked whether this variant might be involved in a generation of a new splice site. Through Human Splicing Finder [19], the splicing motif is not altered due to the nucleotide substitution. Therefore, there is no indication that this variant might have an impact on splicing.

Since three previously reported somatic loss-of-function DOT1L mutations in melanoma affect the methyltransferase activity [7], we aimed to assess whether the DOT1L mutation identified in our family disturbs DOT1L protein function by assessing H3K79 methylation via IHC. A positive and negative controls derived from mouse thymus tissue were included to demonstrate the sensitivity of $\mathrm{H} 3 \mathrm{k} 79$ me antibody. A high percentage $(\sim 80 \%)$ of positive staining nuclei in primary melanoma from II.1 and brain metastasis from III.2 could be observed (see Figure, Supplemental Digital Content 3, http://links.lwrw.com/MR/ A179), indicating that the methyltransferase activity is only marginally, if at all, affected in the tumors.

\section{R409H variant does not significantly affect the ultraviolet sensitivity}

We next determined cell survival upon treatment with $\mathrm{UV}-\mathrm{C}$ radiation through a clonogenic assay with the use of wild-type and CRISPR-Cas9-engineered homozygous DOT1L p.R409H-mutant mESCs. No significant difference could be observed in survival after UV-irradiation between DOT1L p.R409H-mutant and wild-type mESCs while DOT1L p.G165R mutant mESCs, expressing a catalytically inactive DOT1L protein showed reduced survival (Fig. 3a). In mESCs, DOT1L p.R409H mutation did not lead to detectable loss of H3K79 methylation, while methylation was completely lost in the G165R mutant (Fig. 3b).

Fig. 3

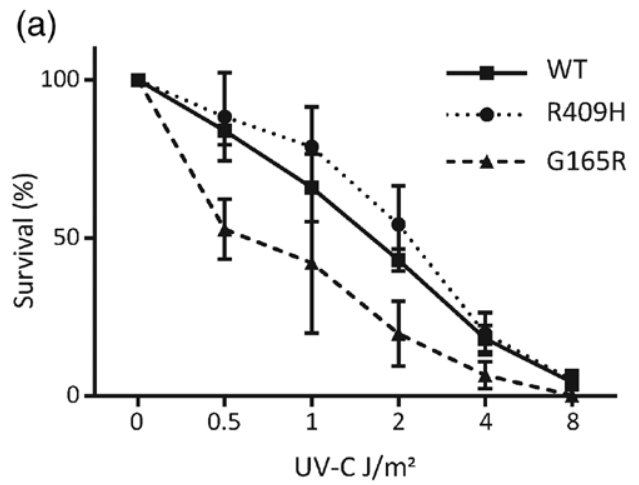

(b)

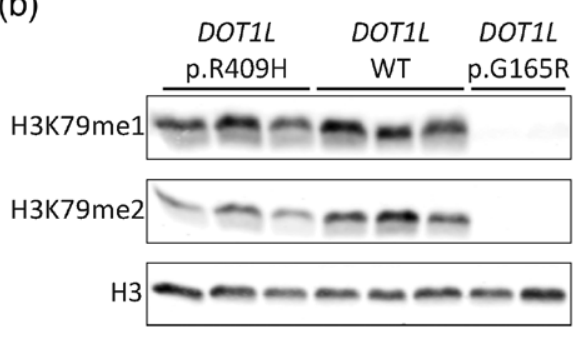

UV-survival assay of WT and DOT1L-mutant mouse embryonic stem cells (mESCs). (a) Colony formation capacity upon UV-C irradiation (dose range: $\left.0.5,1,2,4,8 \mathrm{~J} / \mathrm{m}^{2}\right)$ in wild-type, DOT1L-R409H and DOT1L-G165R mutated mESCs $(\mathrm{n}=3$ independent replicates, error bars represent s.d.) (b) Immunoblot analysis of H3K79me levels in the ESC clones used for UV-survival assay. Each lane shows one independently generated clone as described in materials and methods. UV, ultraviolet. 


\section{Discussion}

Here, we report a novel missense germline mutation in the DOT1L gene shared by four first-degree family members diagnosed with melanoma with an early age of onset. Another variant in SLCO4C1 gene was found to co-segregate with melanoma in the family. However, the lack of evidence in association with cancer or expression in melanocytes did not encourage us to explore it further. On the other hand, DOT1L is a highly evolutionary conserved protein and is the unique histone methyltransferase responsible for mono-, di- and trimethylating the core of histone $\mathrm{H} 3$ on lysine 79 (H3K79) $[5,6,20]$. In addition, DOT1L regulates transcription elongation, establishes cell cycle checkpoints, and maintains genomic stability $[21,22]$. Dysregulation of DOT1L has been associated with a number of cancers either as an oncogene or tumor suppressor gene [20].

The DOT1L protein has been reported to interact with mixed-lineage leukemia (MLL) fusion partners, such as AF4, AF9, AF10 and ENL, leading to H3K79 hypermethylation and transcriptional activation of target genes favoring leukemic transformation [23]. Furthermore, DOT1L was described to interact with c-Myc-p300 complex to activate the epithelial-mesenchymal transition regulators in breast cancer progression [24]. In addition, IL22/STAT3 signaling was reported to increase DOT1L expression, which subsequently increased the transcription of core stem cell genes, enhancing the cancer stemness and colorectal carcinogenesis, correlating with poor patient outcome [25]. In all these studies, DOT1L functions as an oncoprotein.

Recently, DOT1L has been described in colorectal cancer as an important player in DNA double-stand break repair via homologous recombination through $\gamma \mathrm{H} 2 \mathrm{AX}$ phosphorylation [26]. Also in melanoma, a role for DOT1L in DNA damage repair has been envisioned. Three new mutations (M55L, P271L and P505L) in the DOT1L gene that negatively affect the catalytic activity of the methyltransferase were identified [7]. Loss of DOT1L (by silencing or mutation) impaired the DNA damage repair induced by UV-B radiation, thereby promoting melanoma development in vivo. The authors show that DOT1L promotes the assembly of the nucleotide excision repair complex on chromatin by interacting with XPC and stimulating its recruitment to the DNA lesion, but DOT1L is not involved in transcriptional regulation of the DNA repair genes [7]. Therefore, in human melanoma DOT1L seems to behave as a tumor suppressor gene. In mESCs carrying a catalytically inactive G165R mutant, we also observed a protective role of DOT1L against UV radiation.

In our study, the R409H variant in DOT1L gene, which protects melanocytes from the UV-induced transition to melanoma, was identified upon WES of two members of a family with a family history of melanoma. The R409H was confirmed in other two affected family members, therefore co-segregating with melanoma in all four first-degree melanoma-affected family members. Then, we functionally explored this variant but we could neither detect histone methyltransferase activity reduction in melanoma and mESCs nor an effect on UV-induced survival in mESCs. However, it is possible that dynamic changes in or alternative functions of $\mathrm{H} 3 \mathrm{~K} 79 \mathrm{me}$ were missed in the assays used or that the role of R409 in melanocytes is not recapitulated in the cell model used here. Accordingly, two previously reported DOT1L variants (V135A and F243A) hardly showed a decrease of the DOT1L methyltransferase activity [27]. R409 is located in a part of the DOT1L protein that is enriched for positively charged residues [28]. This region contains a nuclear localization signal [29] and is part of a C-terminal extension of the catalytic core of DOT1L that is required for nucleosome binding and DOT1L activity [28]. Furthermore, lysine 410 , adjacent to R409, was identified as a site that can be methylated by SUV39H1, suggesting that the function of this part of DOT1L may be subject to post-translational modifications. SUV39H1 targets RK sites [30], and the R409H mutation disrupts this RK motif. However, very little is known about the interactions between the DOT1L C-terminal extension and the nucleosome. Recent efforts to elucidate the mechanisms of these interactions by determining the structure of DOT1L bound to nucleosomes have not yet revealed the molecular details [31-33]. It has been reported that DOT1L only binds the ubiquitinated nucleosome, which is dependent on H2BK120 monoubiquitination and H2A-H2B acidic path, that subsequently enhances the catalytic function of the methyltransferase DOT1L [27]. However, the lack of unequivocal structural information is most likely caused by the dynamic nature of the interactions between the DOT1L C-terminal extension of the catalytic core and the nucleosome. It could also be possible that the R409H mutation affects a methyltransferase-independent function of DOT1L. For example, budding yeast DOT1L functions as a transcription de-repressor, a histone chaperone and enhances $\mathrm{H} 2 \mathrm{~B}$ ubiquitination all independent of its methyltransferase activity [34-36]. However, in mammalian cells this activity of DOT1L has been shown to be required for several critical functions, including reactivation of repressed genes upon targeting, cycle progression in lung cancer cell lines, and leukaemic transformation in CALM-AF10 MLL-rearranged leukemia [37-39]. Taken together, methyltransferase-independent functions of DOT1L have been reported, but not in mammalian cells. Despite a lack of evidence for a direct functional effect of the $\mathrm{R} 409 \mathrm{H}$ variant, several variants in DOT1L have been observed in independent familial and sporadic melanoma cases. Therefore, our finding reinforces the ones by Zhu et al. [7] and we consider that is worthwhile to investigate the DOT1L variants in future WES and WGS studies involving large 
familial melanoma cohorts, albeit that further functional and structural analyses are required in order to confirm DOT1L to be a melanoma-susceptibility gene.

\section{Acknowledgements}

We would like to acknowledge David Adams' lab (Wellcome Trust Sanger Institute, Wellcome Genome Campus, Cambridge, UK) for sequencing the DNA from two family members through the whole-exome sequencing followed by bioinformatic analysis. We would like to thank Jaap Jansen, Martijn Luijsterburg (Department of Human Genetics, Leiden University Medical Center, Leiden, The Netherlands) and Bart Janssen (GenomeScan B.V., Leiden, The Netherlands) for all scientific discussions and technical expertise and also to the technician Angela Kragten for experimental help, Enno Dreef (Department of Pathology, LUMC) for helping with immunohistochemistry protocol, Wim Zoutman, Coby Out, and Amina Teunisse (Departments of Dermatology and Cell and Chemical Biology) for technical assistance, and Tim Harmsen and Hein te Riele (Department of Tumor Biology and Immunology, NKI, Amsterdam, The Netherlands) for help with the generation of the mutant mESCs using Crispr/Cas9.

This project has received funding from the European Union's Horizon 2020 research and innovation programme under grant agreement No 641458. This study was supported by grants from the Dutch Cancer Society (UL2012-5489 and NKI2014-7232) and The Netherlands Organisation for Scientific Research (NWO-VICI-016.130.627).

\section{Conflicts of interest}

There are no conflicts of interest.

\section{References}

1 Schadendorf D, van Akkooi ACJ, Berking C, Griewank KG, Gutzmer R, Hauschild A, et al. Melanoma. Lancet 2018; 392:971-984.

2 Read J, Wadt KA, Hayward NK. Melanoma genetics. J Med Genet 2016; 53:1-14.

3 Potjer TP, Bollen S, Grimbergen AJEM, van Doorn R, Gruis NA, van Asperen CJ, et al.; Dutch Working Group for Clinical Oncogenetics. Multigene panel sequencing of established and candidate melanoma susceptibility genes in a large cohort of dutch non-CDKN2A/CDK4 melanoma families. Int J Cancer 2019; 144:2453-2464.

4 Potrony M, Badenas C, Aguilera P, Puig-Butille JA, Carrera C, Malvehy J, Puig S. Update in genetic susceptibility in melanoma. Ann Trans/ Med $2015 ; 3: 210$.

5 van Leeuwen F, Gafken PR, Gottschling DE. Dot1p modulates silencing in yeast by methylation of the nucleosome core. Cell 2002; 109:745-756.

6 Nguyen AT, Zhang Y. The diverse functions of dot1 and H3K79 methylation. Genes Dev 2011; 25:1345-1358.

7 Zhu B, Chen S, Wang H, Yin C, Han C, Peng C, et al. The protective role of DOT1L in UV-induced melanomagenesis. Nat Commun 2018; 9:259.

8 Oksenych V, Zhovmer A, Ziani S, Mari PO, Eberova J, Nardo T, et al. Histone methyltransferase DOT1L drives recovery of gene expression after a genotoxic attack. Plos Genet 2013; 9:e1003611.

9 Li H, Durbin R. Fast and accurate short read alignment with burrowswheeler transform. Bioinformatics 2009; 25:1754-1760.

10 Vlaming $\mathrm{H}$, McLean CM, Korthout T, Alemdehy MF, Hendriks S, Lancini $\mathrm{C}$, et al. Conserved crosstalk between histone deacetylation and H3K79 methylation generates DOT1L-dose dependency in HDAC1-deficient thymic lymphoma. EMBO J 2019; 38:e101564.

11 Vlaming $\mathrm{H}$, Molenaar TM, van Welsem T, Poramba-Liyanage DW, Smith DE, Velds $A$, et al. Direct screening for chromatin status on DNA barcodes in yeast delineates the regulome of H3K79 methylation by Dot1. eLife 2016; 5:e18919.

12 Harmsen T, Klaasen S, van de Vrugt H, Te Riele H. DNA mismatch repair and oligonucleotide end-protection promote base-pair substitution distal from a CRISPR/cas9-induced DNA break. Nucleic Acids Res 2018; 46:2945-2955.

13 Mikkaichi T, Suzuki T, Onogawa T, Tanemoto M, Mizutamari H, Okada $\mathrm{M}$, et al. Isolation and characterization of a digoxin transporter and its rat homologue expressed in the kidney. Proc Natl Acad Sci U S A 2004; 101:3569-3574.

14 Ziliak D, O'Donnell PH, Im HK, Gamazon ER, Chen P, Delaney S, et al. Germline polymorphisms discovered via a cell-based, genome-wide approach predict platinum response in head and neck cancers. Trans/ Res 2011; 157:265-272.

15 Guerrero-Preston R, Michailidi C, Marchionni L, Pickering CR, Frederick MJ, Myers JN, et al. Key tumor suppressor genes inactivated by "greater promoter" methylation and somatic mutations in head and neck cancer. Epigenetics 2014; 9:1031-1046.

16 Haltaufderhyde KD, Oancea E. Genome-wide transcriptome analysis of human epidermal melanocytes. Genomics 2014; 104:482-489.

17 Verfaillie A, Imrichova H, Atak ZK, Dewaele M, Rambow F, Hulselmans $\mathrm{G}$, et al. Decoding the regulatory landscape of melanoma reveals TEADS as regulators of the invasive cell state. Nat Commun 2015; $6: 6683$.

18 Liu XS, Genet MD, Haines JE, Mehanna EK, Wu S, Chen HI, et al. ZBTB7A suppresses melanoma metastasis by transcriptionally repressing MCAM. Mol Cancer Res 2015; 13:1206-1217.

19 Desmet FO, Hamroun D, Lalande M, Collod-Béroud G, Claustres M, Béroud C. Human splicing finder: an online bioinformatics tool to predict splicing signals. Nucleic Acids Res 2009; 37:e67.

20 Vlaming $\mathrm{H}$, van Leeuwen $\mathrm{F}$. The upstreams and downstreams of H3K79 methylation by DOT1L. Chromosoma 2016; 125:593-605.

21 Kim W, Choi M, Kim JE. The histone methyltransferase Dot1/DOT1L as a critical regulator of the cell cycle. Cell Cycle 2014; 13:726-738.

22 Wood K, Tellier M, Murphy S. DOT1L and H3K79 methylation in transcription and genomic stability. Biomolecules 2018; 8:E11.

23 Wong M, Polly P, Liu T. The histone methyltransferase DOT1L: regulatory functions and a cancer therapy target. Am J Cancer Res 2015; 5:2823-2837.

24 Cho MH, Park JH, Choi HJ, Park MK, Won HY, Park YJ, et al. DOT1L cooperates with the c-myc-p300 complex to epigenetically derepress $\mathrm{CDH} 1$ transcription factors in breast cancer progression. Nat Commun $2015 ; 6: 7821$.

25 Kryczek I, Lin Y, Nagarsheth N, Peng D, Zhao L, Zhao E, et al. IL-22(+) CD4(+) T cells promote colorectal cancer stemness via STAT3 transcription factor activation and induction of the methyltransferase DOT1L. Immunity 2014; 40:772-784.

26 Kari V, Raul SK, Henck JM, Kitz J, Kramer F, Kosinsky RL, et al. The histone methyltransferase DOT1L is required for proper DNA damage response, DNA repair, and modulates chemotherapy responsiveness. Clin Epigenetics 2019; 11:4.

27 Yao T, Jing W, Hu Z, Tan M, Cao M, Wang Q, et al. Structural basis of the crosstalk between histone $\mathrm{H} 2 \mathrm{~B}$ monoubiquitination and $\mathrm{H} 3$ lysine 79 methylation on nucleosome. Cell research 2019; 29:330-333.

28 Min J, Feng Q, Li Z, Zhang Y, Xu RM. Structure of the catalytic domain of human DOT1L, a non-SET domain nucleosomal histone methyltransferase. Cell 2003; 112:711-723.

29 Reisenauer MR, Wang SW, Xia Y, Zhang W. Dot1a contains three nuclear localization signals and regulates the epithelial $\mathrm{Na}+$ channel $(\mathrm{ENaC})$ at multiple levels. Am J Physiol Renal Physiol 2010; 299:F63-F76.

30 Kudithipudi S, Schuhmacher MK, Kebede AF, Jeltsch A. The SUV39H1 protein lysine methyltransferase methylates chromatin proteins involved in heterochromatin formation and VDJ recombination. ACS Chem Bio/ 2017; 12:958-968.

31 Jang S, Kang C, Yang HS, Jung T, Hebert H, Chung KY, et al. Structural basis of recognition and destabilization of the histone $\mathrm{H} 2 \mathrm{~B}$ ubiquitinated nucleosome by the DOT1L histone H3 Lys79 methyltransferase. Genes Dev 2019; 33:620-625.

32 Worden EJ, Hoffmann NA, Hicks CW, Wolberger C. Mechanism of crosstalk between H2B ubiquitination and $\mathrm{H} 3$ methylation by Dot1L. Cell 2019; 176:1490-1501.e1412. 
33 Anderson CJ, Baird MR, Hsu A, Barbour EH, Koyama Y, Borgnia MJ, et al. Structural basis for recognition of ubiquitylated nucleosome by Dot $1 \mathrm{~L}$ methyltransferase. Cell reports 2019; 26:1681-1690.e1685.

34 Stulemeijer IJ, Pike BL, Faber AW, Verzijlbergen KF, van Welsem T, Frederiks $\mathrm{F}$, et al. Dot1 binding induces chromatin rearrangements by histone methylation-dependent and -independent mechanisms. Epigenetics Chromatin 2011; 4:2.

35 Lee S, Oh S, Jeong K, Jo H, Choi Y, Seo HD, et al. Dot1 regulates nucleosome dynamics by its inherent histone chaperone activity in yeast. Nat Commun 2018; 9:240.

36 van Welsem T, Korthout T, Ekkebus R, Morais D, Molenaar TM, van Harten $\mathrm{K}$, et al. Dot1 promotes $\mathrm{H} 2 \mathrm{~B}$ ubiquitination by a methyltransferase-independent mechanism. Nucleic Acids Res 2018; 46:11251-11261.

37 Cano-Rodriguez D, Gjaltema RA, Jilderda LJ, Jellema P, Dokter-Fokkens J, Ruiters MH, Rots MG. Writing of h3k4me3 overcomes epigenetic silencing in a sustained but context-dependent manner. Nat Commun 2016; 7:12284.

38 Kim W, Kim R, Park G, Park JW, Kim JE. Deficiency of H3K79 histone methyltransferase dot1-like protein (DOT1L) inhibits cell proliferation. J Biol Chem 2012; 287:5588-5599.

39 Okada Y, Jiang Q, Lemieux M, Jeannotte L, Su L, Zhang Y. Leukaemic transformation by CALM-AF10 involves upregulation of hoxa5 by hdot11. Nat Cell Biol 2006; 8:1017-1024. 